\title{
Gambogic acid inhibits spinal cord injury and inflammation through suppressing the p38 and Akt signaling pathways
}

\author{
QIAO FU ${ }^{1,2}$, CHAOJIAN $\mathrm{LI}^{2}$ and LEHUA YU ${ }^{1}$ \\ ${ }^{1}$ Department of Rehabilitation Medicine and Physical Therapy, The Second Affiliated Hospital of \\ Chongqing Medical University, Chongqing 400010; ${ }^{2}$ Department of Rehabilitation Medicine, \\ Hainan General Hospital, Haikou, Hainan 570311, P.R. China
}

Received November 5, 2016; Accepted August 31, 2017

DOI: $10.3892 / \mathrm{mmr} .2017 .8026$

\begin{abstract}
Gamboge is the dry resin secreted by Garcinia hanburyi Hook.f, with the function of promoting blood circulation, detoxification, hemostasis and killing insects, used for the treatment of cancer, brain edema and other diseases. Gambogic acid is the main effective constituent of Gamboge. The present study investigated the protective effects of gambogic acid on spinal cord injury (SCI) and its anti-inflammatory mechanism in an SCI model in vivo. Basso, Beattie and Bresnahan (BBB) testing was used to detect the protective effects of gambogic acid on nerve function of SCI rats. The water content of the spinal cord was used to analyze the protective effects of gambogic acid on the damage of SCI. Treatment with gambogic acid effectively improved BBB scores and inhibited water content of the spinal cord in SCI rats. Also, gambogic acid significantly reduced inflammatory cytokines levels of [tumor necrosis factor- $\alpha$, interleukin (IL)-6, IL-12 and IL-1 $\beta$ ] and oxidative stress (malondialdehyde, superoxide dismutase, glutathione and glutathione-peroxidase) factors, and suppressed receptor activator of nuclear factor $\kappa \mathrm{B}$ ligand, phosphorylated p38 protein expression and toll-like receptor $4 /$ nuclear factor- $\kappa \mathrm{B}$ pathway activation, and increased phosphatidylinositol 3-kinase/protein kinase B (Akt) pathway activation in SCI rats. These results provide evidence that gambogic acid inhibits SCI and inflammation through suppressing the p38 and Akt signaling pathways.
\end{abstract}

\section{Introduction}

Spinal cord injury (SCI) refers to different degrees of injury to the spinal cord due to a variety of external direct or indirect

Correspondence to: Dr Lehua Yu, Department of Rehabilitation Medicine and Physical Therapy, The Second Affiliated Hospital of Chongqing Medical University, 76 Linjiangmen Road, Chongqing 400010, P.R. China

E-mail: uhnz8178705@126.com

Key words: gambogic acid, spinal cord injury, p38, protein kinase $\mathrm{B}$, nuclear factor- $\kappa \mathrm{B}$ traumas (1). SCI usually leads to severe consequences, such as loss of sensation at the area of injury, partial dysfunction of limbs (2). With the rapid development of modern science, technology, industry and transportation, the incidence of SCI has increased significantly; therefore, the epidemiological investigations about SCI are also increasing. Most epidemiological investigations demonstrate that the overall incidence rate of SCI increases annually (3). Additionally, previous age spectrum studies on SCI revealed that SCI mostly occurs in young adults (especially due to traffic- and factory-associated accidents) (2). In addition, SCI has very high disability rate, which brings serious economic and emotional burdens to individuals, families and society (4).

SCI is divided into two stages: One is the injury to specific regions of the spinal cord caused by the initial trauma; the other is a secondary injury involving a series of biochemical, molecular and cellular changes (5). SCI leads to a systemic inflammatory response, and inflammatory cells invade other remote organs such as the liver, lungs and kidneys, causing damage to these organs. Inflammation and oxidative stress are two main factors of SCI, and enhance the release of excitatory amino acids in the process of cell apoptosis, to upregulate the generation of reactive oxygen species and lipid peroxide, causing SCI-induced secondary injury (6).

Protein kinase B (Akt) is a key kinase regulating the proliferation, differentiation, apoptosis and death of cells. Akt is activated by translocation to the inner surface of cell membranes and subsequent phosphorylation (7). Phosphatidylinositol 3-kinase (PI3K) is activated by the phosphorylation of the third hydroxyl group on its inositol ring, which further phosphorylates inositol in the cell membrane to translocate serine/threonine protein kinase (8). Previous studies have indicated that the activation of Akt protects nerves by inhibiting the apoptosis of nervous cells, reducing the generation of oxygen free radicals and suppressing the inflammatory reaction when SCI occurs $(7,9)$.

It is understood that the mitogen activated protein kinase (MAPK) signaling pathway is an important intracellular signal transduction pathway. The MAPK family has three subfamily pathways, including extracellular regulated protein kinase (ERK1/2), c-Jun N-terminal kinase (JNK) and P38 MAPK (10). When these MAPK pathways are activated by a variety of factors such as lipopolysaccharide, they will produce 
a large number of inflammatory mediators through complex intracellular signal transduction, leading to inflammation and promoting its development (10). Elevated phosphorylation is a sign of activation of the ERK1/2, JNK and MAPK P38 signaling pathway (11).

Gamboge is the dry resin secreted by Garcinia hanburyi Hook.f. Gambogic acid, the main active constituent of the resin produced by Garcinia hanburyi Hook.f, is a type of natural small-molecule Xanthone drug (Fig. 1) (12). Gambogic acid has long been used as anti-inflammatory, detoxification and insecticidal drug in Southeast Asia (13). The present study aimed to explore the protective effects of gambogic acid on SCI, and its anti-inflammation mechanism in an SCI model in vivo.

\section{Materials and methods}

Animals and in vivo treatment. Male Sprague-Dawley rats (weight, 200-220 g; n=24) were purchased from the Animal Experiment Centre of Chongqing Medical University (Chongqing, China) housed in a room controlled for temperature $\left(23 \pm 3^{\circ} \mathrm{C}\right)$ and relative humidity (40-60\%), and had free access to food and water. Animal care and study protocols were carried out in accordance with the guidelines of the Institutional Animal Care and Use Committee of the Second Affiliated Hospital of Chongqing Medical University (Chongqing, China). Ethical approval was received from the medical ethics committee of Hainan General Hospital (Haikou, China). All rats were randomly assigned into three groups ( $\mathrm{n}=8 /$ group): Sham-operated (sham), SCI model (SCI) and gambogic acid (GA).

An SCI model was induced as previously described (14). In the SCI model and GA groups, rats were anesthetized with pentobarbital sodium $(35 \mathrm{mg} / \mathrm{kg}$, intraperitoneally; Sigma-Aldrich; Merck KGaA, Darmstadt, Germany), and laminectomy was performed at the T9-T11 level in every rat, which exposed the underlying cord. A weight-drop apparatus was used to induce spinal cord contusion, at a height of $80 \mathrm{~mm}$ dropped onto the exposed cord, representing moderate SCI. The skin and musculature were sutured. In the sham group, rats underwent a sham operation group without inducing SCI. In the GA group, rats underwent SCI followed by $2 \mathrm{mg} / \mathrm{kg} / \mathrm{three}$ days GA treatment for 10 weeks.

Behavioral testing and water content of the spinal cord. All rats were assessed with the Basso, Beattie and Bresnahan (BBB) scale test (15). A score of 0 indicated complete hind limb paralysis, and a score of 21 denoted completely normal locomotor function. After rats were anesthetized with $10 \%$ chloral hydrate $(3.5 \mathrm{mg} / \mathrm{kg}$, intraperitoneally), rats were sacrificed using decollation and spinal cord tissue samples were weighed to obtain the wet weight $(\mathrm{g})$, then dried at $68^{\circ} \mathrm{C}$ for $48 \mathrm{~h}$ to obtain the dry weight $(\mathrm{g})$. Water content of spinal cord $(\%)=$ dry weight/wet weight $\times 100 \%$.

Determination of inflammatory cytokines, oxidative stress and caspase-3 activity. After rats were anesthetized with $10 \%$ chloral hydrate $(3.5 \mathrm{mg} / \mathrm{kg}$, intraperitoneally), and peripheral blood was acquired from the eye socket. Following sacrifice by decollation, serum was acquired

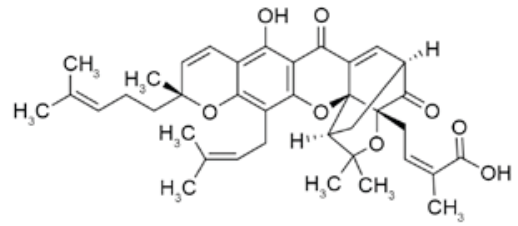

Figure 1. Chemical structure of gambogic acid.

after centrifuging blood at $2,000 \mathrm{x}$ g for $10 \mathrm{~min}$ at $4^{\circ} \mathrm{C}$. Inflammatory cytokines [tumor necrosis factor (TNF)- $\alpha$ (cat. no. PT516; Beyotime Institute of Biotechnology, Haimen, China), interleukin (IL)-6 (cat. no. PI328; Beyotime Institute of Biotechnology), IL-12 (cat. no. H010; Beyotime Institute of Biotechnology) and IL-1 $\beta$ (cat. no. PI303, Beyotime Institute of Biotechnology)] and oxidative stress factors [malondialdehyde (MDA; cat. no. S0131; Beyotime Institute of Biotechnology), superoxide dismutase (SOD; cat. no. S0101; Beyotime Institute of Biotechnology), glutathione (GSH; cat. no. S0052; Beyotime Institute of Biotechnology) and glutathione peroxidase (GSH-PX; cat. no. S0058; Beyotime Institute of Biotechnology)] were measured using ELISA kits using fluorescence microplate reader (Model 680, Bio-Rad Laboratories, Inc., Hercules, CA, USA) at a wavelength of $450 \mathrm{~nm}$. Caspase-3 activity (cat. no. C1116; Beyotime Institute of Biotechnology) was measured using an ELISA kit using a fluorescence microplate reader at a wavelength of $405 \mathrm{~nm}$.

Western blot analysis. Radioimmunoprecipitation assay lysis buffer containing PMSF was added into spinal cord tissue samples for $30 \mathrm{~min}$ on ice, and the supernatant was harvested by centrifugation at $4^{\circ} \mathrm{C}$ at $8,000 \mathrm{x} \mathrm{g}$ for $10 \mathrm{~min}$. The protein concentration was detected using a bicinchoninic acid protein assay kit. Proteins $(50 \mu \mathrm{g})$ were separated by 6-10\% SDS-PAGE and transferred to polyvinylidene difluoride membranes. The membranes were blocked in TBS with Tween 20 containing 5\% nonfat dry milk for $1 \mathrm{~h}$ at $37^{\circ} \mathrm{C}$. They were then incubated with the following primary antibodies: Anti-receptor activator of nuclear factor- $\kappa \mathrm{B}$ ligand (RANKL; cat. no. sc-9073; 1:500), anti-toll-like receptor 4 (TLR4; cat. no. sc-10741; 1:500), anti-nuclear factor (NF)- $\mathrm{B}$ (cat. no. sc-298, 1:500), anti-phosphorylated (p)-NF- $\mathrm{B}$ (cat. no. sc-136548; 1:500), anti-p-p38 (cat. no. sc-7975-R, 1:500), anti-p38 (cat. no. sc-7149; 1:500), anti-PI3K (cat. no. sc-7174; 1:500), anti-Akt (cat. no. sc-8312; 1:500) and anti-GADPH (cat. no. sc-25778; 1:500; all Santa Cruz Biotechnology, Inc.) at $4{ }^{\circ} \mathrm{C}$ overnight. After being washed three times with TBST for $15 \mathrm{~min}$, the membranes were incubated with a horseradish peroxidase-conjugated anti-mouse IgG secondary antibody (cat. no. sc-2030; 1:5,000; Santa Cruz Biotechnology, Inc., Dallas, TX, USA) for $1 \mathrm{~h}$ at $37^{\circ} \mathrm{C}$ and visualized by BeyoECL Plus (cat. no. P0018A; Beyotime Institute of Biotechnology).

Statistical analysis. Data are presented as the mean \pm standard error and analyzed using SPSS 17.0 software (SPSS, Inc., Chicago, IL, USA). Comparisons of data between groups were performed by one-way analysis of variance followed by Duncan's multiple range test. $\mathrm{P}<0.05$ was considered to indicate a statistically significant difference. 

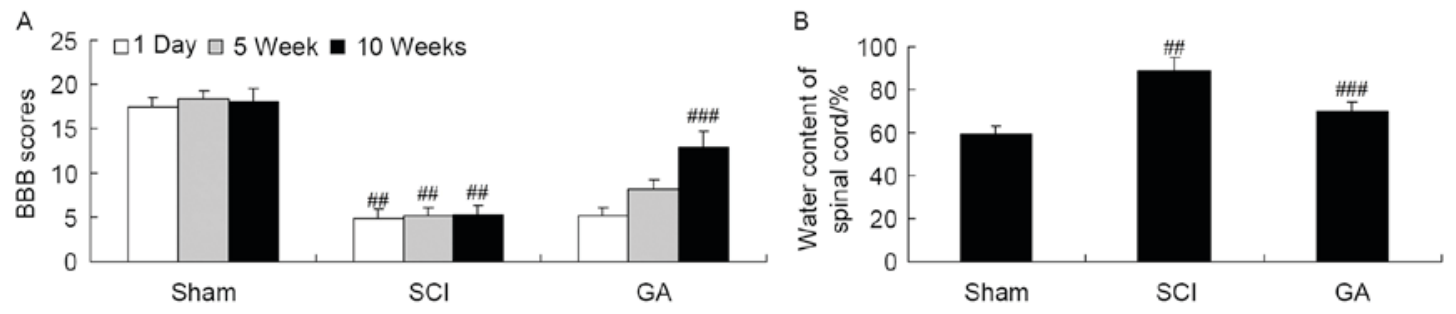

Figure 2. Gambogic acid effects on BBB scores and water content of the spinal cord in SCI rats. Gambogic acid effects on (A) BBB scores and (B) water content of the spinal cord in SCI rats. Data are presented as the mean \pm standard error. ${ }^{\# \#} \mathrm{P}<0.05$ vs. sham group, ${ }^{\# \# \#} \mathrm{P}<0.05$ vs. SCI model group. SCI, spinal cord injury; GA, gambogic acid; BBB, Basso, Beattie and Bresnahan.
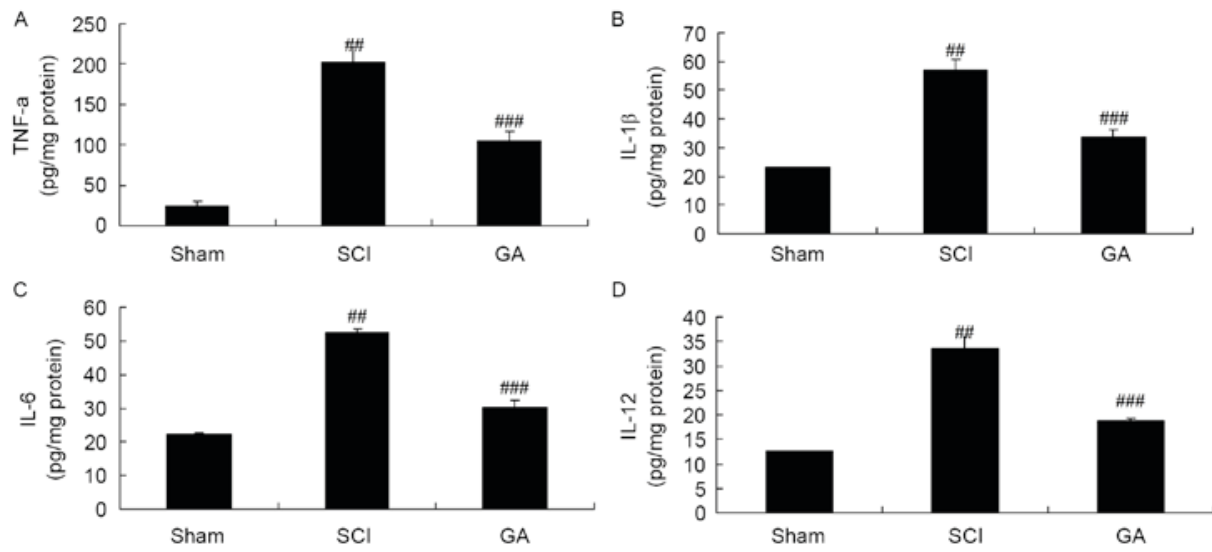

Figure 3. Gambogic acid effects on inflammatory cytokines in SCI rats. Gambogic acid effects on (A) TNF- $\alpha$, (B) IL-1 $\beta$, (C) IL-6 and (D) IL-12 activities. Data are presented as the mean \pm standard error. ${ }^{\# \#} \mathrm{P}<0.05$ vs. sham group, ${ }^{\# \# \#} \mathrm{P}<0.05$ vs. SCI model group. SCI, spinal cord injury; GA, gambogic acid; TNF, tumor necrosis factor; IL, interleukin.
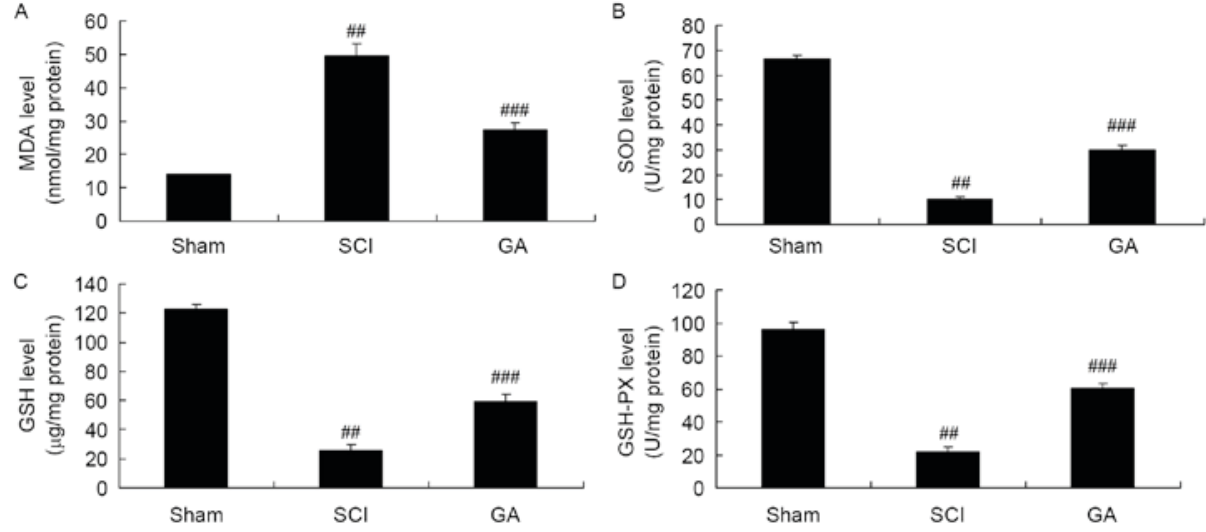

Figure 4. Gambogic acid effects on oxidative stress in SCI rats. Gambogic acid effects on (A) MDA, (B) SOD, (C) GSH and (D) GSH-PX. Data are presented as the mean \pm standard error. ${ }^{\# \#} \mathrm{P}<0.05$ vs. sham group, ${ }^{\# \# \#} \mathrm{P}<0.05$ vs. SCI model group. SCI, spinal cord injury; GA, gambogic acid; MDA, malondialdehyde; SOD, superoxide dismutase; GSH, glutathione; PX, peroxidase.

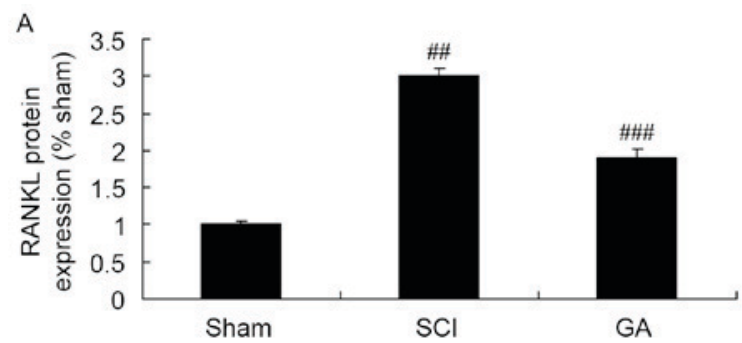

B

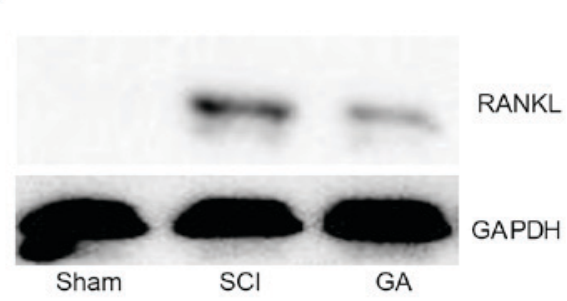

Figure 5. Gambogic acid effects on RANKL protein expression in SCI rats. (A) Quantification and (B) representative western blot images of the effects of gambogic acid on RANKL protein expression. GAPDH served as an internal control. Data are presented as the mean \pm standard error. ${ }^{\sharp \#} \mathrm{P}<0.05$ vs. sham group,

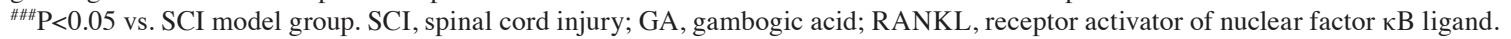




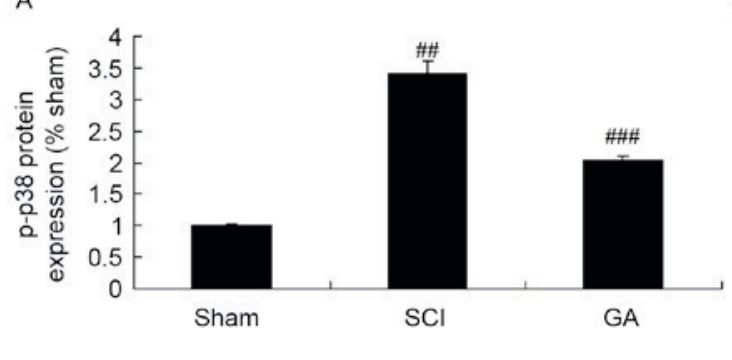

B

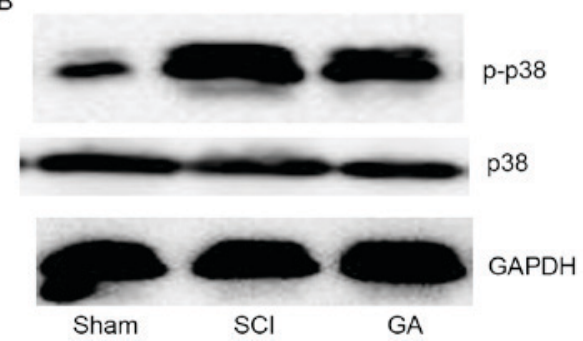

Figure 6. Gambogic acid effects on p-p38 protein expression in SCI rats. (A) Quantification and (B) representative western blot images of the effects of gambogic acid on p-p38 protein expression. GAPDH served as an internal control. Data are presented as the mean \pm standard error. ${ }^{\# \# ~} \mathrm{P}<0.05$ vs. sham group,



A

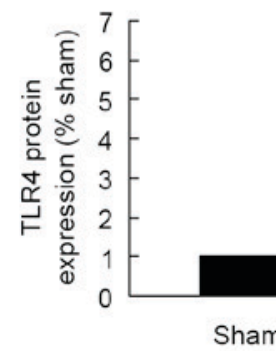

C

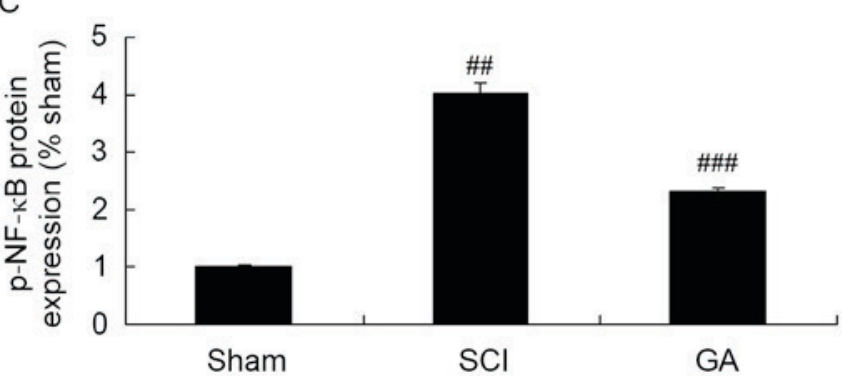

GA
B

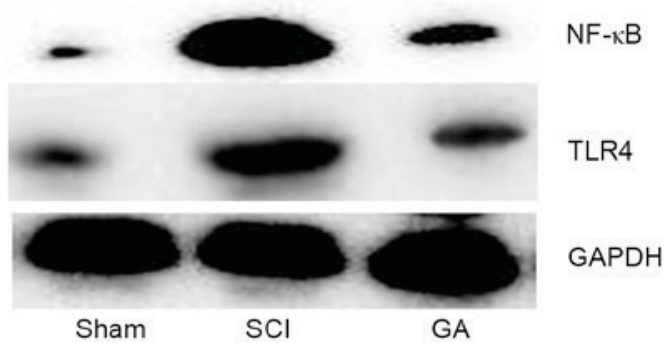

$p-N F-\kappa B$

F-kB

\#\#\#

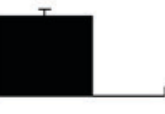

GA

Sham

$\mathrm{SCl}$

GA

Figure 7. Gambogic acid effects on TLR4/NF- $\mathrm{BB}$ protein expression in SCI rats. (A) Quantification of TLR4 protein expression. (B) Representative western

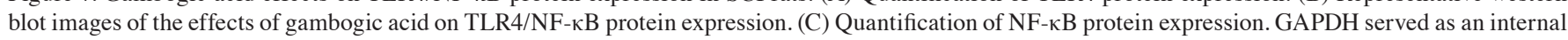
control. Data are presented as the mean \pm standard error. ${ }^{\# \#} \mathrm{P}<0.05$ vs. sham group, ${ }^{\# \# \#} \mathrm{P}<0.05$ vs. SCI model group. SCI, spinal cord injury; GA, gambogic acid; TLR4, toll-like receptor 4; NF, nuclear factor; p-phosphorylated.

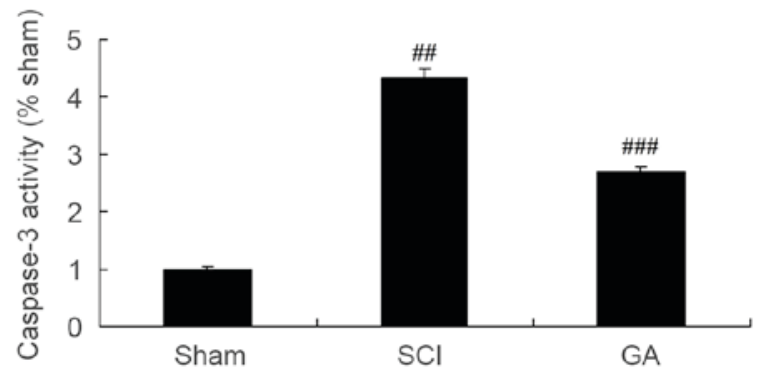

Figure 8. Gambogic acid effects on caspase-3 activity in SCI rats. Data are presented as the mean \pm standard error. ${ }^{\# \#} \mathrm{P}<0.05$ vs. sham group, ${ }^{\# \# \#} \mathrm{P}<0.05$ vs. SCI model group. SCI, spinal cord injury; GA, gambogic acid.

\section{Results}

Gambogic acid effects on BBB scores and the water content of the spinal cord in SCI rats. Firstly, SCI rats were used to investigate the neuroprotective effects of gambogic acid.
There was a significant inhibition of BBB scores (Fig. 2A) and increase of water content of the spinal cord (Fig. 2B) in the SCI model group, compared with the sham group. Following treatment with gambogic acid for 10 weeks, BBB scores were significantly increased and water content of spinal cord was significantly decreased in SCI rats by gambogic acid treatment (Fig. 2).

Gambogic acid effects on inflammatory cytokines in SCI rats. In the SCI model group, TNF- $\alpha$, IL-6, IL-12 and IL-1 $\beta$ levels were significantly enhanced, compared with the sham group (Fig. 3). Treatment with gambogic acid significantly inhibited TNF- $\alpha$, IL-6, IL-12 and IL-1 $\beta$ levels in SCI rats, compared with the SCI model group (Fig. 3).

Gambogic acid effects on oxidative stress in SCI rats. Next, there was a significant increase of MDA activity and inhibition of SOD, GSH and GSH-PX activities in the SCI model group, compared with sham group (Fig. 4). Treatment with gambogic acid significantly reduced MDA activity and increased SOD, 
A

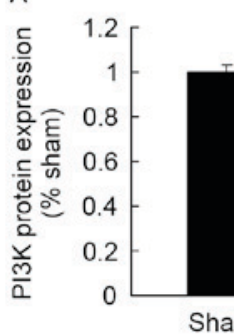

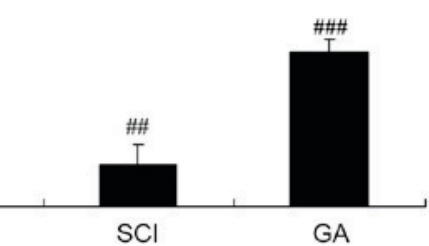

GA



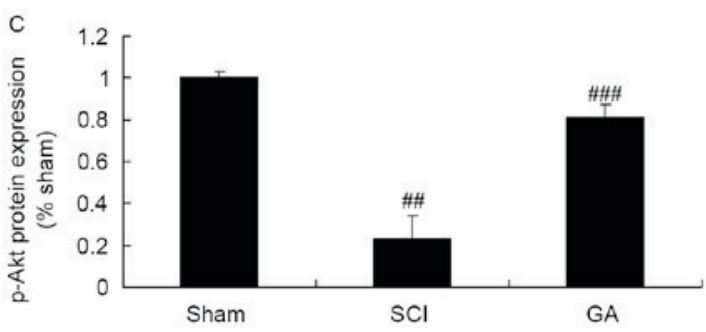

Figure 9. Gambogic acid effects on PI3K/Akt protein expression in SCI rats. (A) Quantification of PI3K protein expression. (B) Representative western blot images of the effects of gambogic acid on PI3K/Akt protein expression. (C) Quantification of p-Akt protein expression. GAPDH served as an internal control. Data are presented as the mean \pm standard error. ${ }^{\# \#} \mathrm{P}<0.05$ vs. sham group, ${ }^{\# \# \#} \mathrm{P}<0.05$ vs. SCI model group. SCI, spinal cord injury; GA, gambogic acid; Akt, protein kinase B; p-, phosphorylated; PI3K, phosphatidylinositol 3-kinase.

GSH and GSH-PX activity inhibition in the SCI rats, compared with the SCI model (Fig. 4).

Gambogic acid effects on RANKL protein expression in SCI rats. Western blotting was used to determine RANKL protein expression in SCI rats. In the SCI model group, there was a significant increase of RANKL protein expression, compared with the sham group (Fig. 5). As presented in Fig. 5, treatment with gambogic acid significantly suppressed increase of RANKL protein expression in SCI rats, compared with the SCI model group.

Gambogic acid effects on $p$-p38 protein expression in $S C I$ rats. The SCI model group exhibited a significant increase of p-p38 protein expression in SCI model rats, compared with the sham group (Fig. 6). Treatment with gambogic acid significantly suppressed p-p38 protein expression in SCI rats, compared with the SCI model group (Fig. 6).

Gambogic acid effects on TLR $4 / N F-\kappa B$ protein expression in SCI rats. To explore the anti-inflammation mechanism of gambogic acid in SCI, TLR4/NF- $\mathrm{KB}$ protein expression were measured using western blot analysis. The results of western blot analysis demonstrated a significant increase of TLR4 and p-NF- $\mathrm{KB}$ protein expression in the SCI model group, compared with the sham group (Fig. 7). However, gambogic acid significantly suppressed TLR4 and p-NF- $\mathrm{\kappa B}$ protein expression in SCI rats, compared with the SCI model group (Fig. 7).

Gambogic acid effects on caspase-3 activity in SCI rats. As presented in Fig. 8, a significant increase of caspase-3 activity was observed in the SCI model group, compared with the sham group. After treatment with gambogic acid for 10 weeks, caspase-3 activity was significantly inhibited in SCI rats, compared with the SCI model group (Fig. 8).
Gambogic acid effects on PI3K/Akt protein expression in SCI rats. To investigate the anti-apoptosis mechanism of gambogic acid in SCI, PI3K/Akt protein expression was measured using western blot analysis. As presented in Fig. 9, PI3K and p-Akt protein expression levels were significantly inhibited in the SCI model group, compared with the sham group. Gambogic acid significantly induced PI3K and p-Akt protein expression levels in SCI rats, compared with the SCI model group (Fig. 9).

\section{Discussion}

SCI, a central nervous system disorder featuring the loss of the sensation, movement and reflexes, and dysfunction of sphincter under the area of injury, results from vertebral fracture and compression to the spinal cord due to mechanical injury (2). Common causes of SCI include traffic accidents, sports injuries, bullet injury, falls and natural disasters (16). At present, $\mathrm{SCI}$ is characterized by high incidence, high disability rate and a huge economic burden (17). The treatment of SCI is a hotspot and difficult problem in clinical research (17). The results of the present study demonstrated that gambogic acid increased BBB scores and decreased the water content of the spinal cord in SCI rats.

Normally, the spinal cord forms an immune privileged region through the blood-brain-barrier, preventing invasion by antibodies and immune cells. However, when the spinal cord is injured, the blood-brain-barrier will be destroyed, thereby leading to an intensive local immune inflammatory reaction (18). ILs, TNF- $\alpha$ inflammatory factors and chemical factors will be released to assist to repair the body. However, excessive activation of the inflammatory response also damages normal cells (19). Therefore, it is important to study interventions in the inflammatory response, as appropriately controlling the inflammatory response can prevent further damage to the body, providing novel ideas for the treatment of SCI (20). The present study confirmed that gambogic acid 
significantly reduced the levels of inflammatory cytokines (TNF- $\alpha$, IL-6, IL-12 and IL-1 $\beta$ ), oxidative stress factors (MDA, SOD, GSH and GSH-PX) in SCI rats. Cascão et al (21) reported that potent anti-inflammatory effects of gambogic acid suppressed antigen-induced arthritis.

The NF- $\mathrm{KB}$ signaling pathway serves an important role in the incidence and development of SCI, and has many downstream targets. I $\mathrm{B}$ can be phosphorylated by a variety of inflammatory factors, and the subunit p65 is activated and then enters into the nucleus (22). The activation of subunit p65 enhances the transcription and expression of IL-1B and TNF- $\alpha$, which can activate the NF- $\kappa$ B signaling pathway again. The MAPK signaling pathway is widely distributed in mammals. NF- $\mathrm{kB}$, one of the targets of the MAPK signaling pathway, is involved in inflammatory responses, as well as the proliferation, differentiation and apoptosis of cells (23). The MAPK pathway is highly conserved, involving three types of kinases, which can be triggered by a variety of factors such as growth factors, cytokines, hormones and proteins (24). Through the cascade reaction, the MAPK pathway alters the secretion of certain cytokines to initiate their biological functions, so as to influence the development and prognosis of inflammatory responses (24). The activation of the MAPK signaling pathway may increase production of cytokines such as TNF- $\alpha$, IL- 6 , IL-12 and IL- $1 \beta$, leading to inflammation and immune responses (25). In the present study, it was demonstrated that gambogic acid significantly suppressed TLR4 and NF- $\mathrm{kB}$ protein expression and induced the p-p38 MAPK signaling pathway in SCI rats.

The mechanism of the differentiation of human bone marrow stromal cells (hBMSCs) into neurons and glial cells in vitro induced by RANKL needs further investigation, and it may be due to the following mechanisms: i) RANKL binds to and activates RANK; ii) RANK interacts with TNF receptor-associated factors to activate NF- $\kappa B$; iii) NF- $\mathrm{NB}$ enters into the nucleus from the cytoplasm rapidly, to bind to the $\kappa \mathrm{B}$ site of target genes and induce the transcription of corresponding target genes; iv) the expression of transcription factors involved in the differentiation of neural cells are up-regulated when selective genes are switched on or off, to pass signals required in the differentiation of neural cells and thus inducing the differentiation of hBMSCs into neural cells $(26,27)$. In the present study, gambogic acid significantly suppressed the increase of RANKL protein expression in SCI rats. Pandey et al (13) demonstrated that gambogic acid inhibits multiple myeloma mediated osteoclastogenesis through the NF- $\kappa \mathrm{B}$ and RANKL signaling pathways.

Akt is the central point of the cellular signal transduction pathway, and is usually stimulated by cytokines and growth factors to pass signals, thereby inducing changes under stress (28). Akt serves an important role in the metabolism, survival, proliferation, differentiation and other key biological functions of cells (9). In addition, Akt serves as an important central control factor in regulating the survival of neurons in the central nervous system, and its signal transduction participates in the survival, development, proliferation, differentiation, axonal growth and synaptic plasticity of neurons (29). In this study, it was demonstrated that gambogic acid significantly inhibited caspase- 3 activity and induced $\mathrm{PI} 3 \mathrm{~K}$ and p-Akt protein expression levels in SCI rats, which demonstrated that the PI3K/Akt signaling pathway may be involved in the anti-apoptosis mechanism of gambogic acid in SCI. Ma et al (12) demonstrated that Gambogic acid inhibits osteoclast formation via RANKL, p38 and Akt.

In conclusion, the results of the present study revealed that gambogic acid inhibits SCI and SCI-induced inflammation, oxidative stress and apoptosis through the TLR4/NF- $\mathrm{KB}$ protein/p38 and Akt signaling pathways. Thus, gambogic acid may be a promising approach to treat SCI in the future.

\section{References}

1. Raithatha R, Carrico C, Powell ES, Westgate PM, Chelette Ii KC, Lee K, Dunsmore L, Salles S and Sawaki L: Non-invasive brain stimulation and robot-assisted gait training after incomplete spinal cord injury: A randomized pilot study. Neuro Rehabilitation 38: 15-25, 2016.

2. Ness LL and Field-Fote EC: Effect of whole-body vibration on quadriceps spasticity in individuals with spastic hypertonia due to spinal cord injury. Restor Neurol Neurosci 27: 621-631, 2009.

3. Biglari B, vd Linden PH, Simon A, Aytac S, Gerner HJ and Moghaddam A: Use of Medihoney as a non-surgical therapy for chronic pressure ulcers in patients with spinal cord injury. Spinal Cord 50: 165-169, 2012.

4. Nussbaum EL, Flett H, Hitzig SL, McGillivray C, Leber D, Morris $\mathrm{H}$ and Jing F: Ultraviolet-C irradiation in the management of pressure ulcers in people with spinal cord injury: A randomized, placebo-controlled trial. Arch Phys Med Rehabil 94: 650-659, 2013

5. Cooney SJ, Zhao Y and Byrnes KR: Characterization of the expression and inflammatory activity of NADPH oxidase after spinal cord injury. Free Radic Res 48: 929-939, 2014.

6. Lu T, Zhang C, Chai M and An Y: Isoquercetin ameliorates tunicamycin-induced apoptosis in rat dorsal root ganglion neurons via suppressing ROS-dependent endoplasmic reticulum stress. Biomed Pharmacother 80: 343-351, 2016.

7. Jung SY, Kim DY, Yune TY, Shin DH, Baek SB and Kim CJ: Treadmill exercise reduces spinal cord injury-induced apoptosis by activating the PI3K/Akt pathway in rats. Exp Ther Med 7: 587-593, 2014.

8. Zhang P and Ma X: Effect of rutin on spinal cord injury through inhibition of the expression of MIP-2 and activation of MMP-9, and downregulation of Akt phosphorylation. Mol Med Rep 12: 7554-7560, 2015.

9. Kim JH, Kim SH, Cho SR, Lee JY, Kim JH, Baek A and Jung HS: The modulation of neurotrophin and epigenetic regulators: Implication for astrocyte proliferation and neuronal cell apoptosis after spinal cord injury. Ann Rehabil Med 40: 559-567, 2016.

10. Cao J, Wang JS, Ren XH and Zang WD: Spinal sample showing p-JNK and P38 associated with the pain signaling transduction of glial cell in neuropathic pain. Spinal Cord 53: 92-97, 2015.

11. Malon JT and Cao L: Calcitonin gene-related peptide contributes to peripheral nerve injury-induced mechanical hypersensitivity through CCL5 and p38 pathways. J Neuroimmunol 297: 68-75, 2016.

12. Ma J, Ma Y, Liu X, Chen S, Liu C, Qin A and Fan S: Gambogic acid inhibits osteoclast formation and ovariectomy-induced osteoporosis by suppressing the JNK, p38 and Akt signalling pathways. Biochem J 469: 399-408, 2015.

13. Pandey MK, Karelia D and Amin SG: Gambogic acid and its role in chronic diseases. Adv Exp Med Biol 928: 375-395, 2016.

14. Wu Y, Streijger F, Wang Y, Lin G, Christie S, Mac-Thiong JM, Parent S, Bailey CS, Paquette S, Boyd MC, et al: Parallel metabolomic profiling of cerebrospinal fluid and serum for identifying biomarkers of injury severity after acute human spinal cord injury. Sci Rep 6: 38718, 2016.

15. Zhang H, Wang L, Wen S, Xiang Q, Xiang X, Xu C, Wan Y, Wang J, Li B, Wan Y, et al: Magnetic resonance imaging tracking and assessing repair function of the bone marrow mesenchymal stem cells transplantation in a rat model of spinal cord injury. Oncotarget 8: 58985-58999, 2017.

16. Giuliano F, Sanchez-Ramos A, Löchner-Ernst D, Del Popolo G, Cruz N, Leriche A, Lombardi G, Reichert S, Dahl P, Elion-Mboussa A and Casariego J: Efficacy and safety of tadalafil in men with erectile dysfunction following spinal cord injury. Arch Neurol 64: 1584-1592, 2007. 
17. Derakhshanrad N, Saberi H, Yekaninejad MS, Eskandari G, Mardani A, Rahdari F and Meybodi KT: Safety of granulocyte colony-stimulating factor (G-CSF) administration for postrehabilitated motor complete spinal cord injury patients: An open-label, phase I study. Cell Transplant 22 (Suppl 1): S139-S146, 2013.

18. Segal JL, Gonzales E, Yousefi S, Jamshidipour L and Brunnemann SR: Circulating levels of IL-2R, ICAM-1, and IL-6 in spinal cord injuries. Arch Phys Med Rehabil 78: 44-47, 1997.

19. Badner A, Vawda R, Laliberte A, Hong J, Mikhail M, Jose A, Dragas R and Fehlings M: Early intravenous delivery of human brain stromal cells modulates systemic inflammation and leads to vasoprotection in traumatic spinal cord injury. Stem Cells Transl Med 5: 991-1003, 2016.

20. Khayrullina G, Bermudez S and Byrnes KR: Inhibition of NOX2 reduces locomotor impairment, inflammation, and oxidative stress after spinal cord injury. J Neuroinflammation 12: 172, 2015.

21. Cascão R, Vidal B, Raquel H, Neves-Costa A, Figueiredo N, Gupta V, Fonseca JE and Moita LF: Potent anti-inflammatory and antiproliferative effects of gambogic acid in a rat model of antigen-induced arthritis. Mediators Inflamm 2014: 195327, 2014

22. Yuan B, Liu D and Liu X: Spinal cord stimulation exerts analgesia effects in chronic constriction injury rats via suppression of the TLR4/NF- $\kappa$ B pathway. Neurosci Lett 581: 63-68, 2014.

23. Pratheeshkumar P, Son YO, Wang X, Divya SP, Joseph B, Hitron JA, Wang L, Kim D, Yin Y, Roy RV, et al: Cyanidin-3-glucoside inhibits UVB-induced oxidative damage and inflammation by regulating MAP kinase and NF- $\mathrm{KB}$ signaling pathways in SKH-1 hairless mice skin. Toxicol Appl Pharmacol 280: 127-137, 2014.
24. Luo Y, Fu C, Wang Z, Zhang Z, Wang H and Liu Y: Asiaticoside attenuates the effects of spinal cord injury through antioxidant and anti-inflammatory effects, and inhibition of the p38-MAPK mechanism. Mol Med Rep 12: 8294-8300, 2015.

25. Horvath RJ, Landry RP, Romero-Sandoval EA and DeLeo JA: Morphine tolerance attenuates the resolution of postoperative pain and enhances spinal microglial p38 and extracellular receptor kinase phosphorylation. Neuroscience 169: 843-854, 2010.

26. Liu HJ, Yan H, Yan J, Li H, Chen L, Han LR and Yang XF: Substance $\mathrm{P}$ promotes the proliferation, but inhibits differentiation and mineralization of osteoblasts from rats with spinal cord injury via RANKL/OPG system. PLoS One 11: e0165063, 2016.

27. Maïmoun L, Couret I, Mariano-Goulart D, Dupuy AM, Micallef JP, Peruchon E, Ohanna F, Cristol JP, Rossi M and Leroux JL: Changes in osteoprotegerin/RANKL system, bone mineral density, and bone biochemicals markers in patients with recent spinal cord injury. Calcif Tissue Int 76: 404-411, 2005.

28. Zhang P, Zhang L, Zhu L, Chen F, Zhou S, Tian T, Zhang Y, Jiang X, Li X, Zhang C, et al: The change tendency of PI3K/Akt pathway after spinal cord injury. Am J Transl Res 7: 2223-2232, 2015.

29. Stover J and Nagatomi J: Cyclic pressure stimulates DNA synthesis through the PI3K/Akt signaling pathway in rat bladder smooth muscle cells. Ann Biomed Eng 35: 1585-1594, 2007. 